Publ. RIMS, Kyoto Univ.

12 Suppl. (1977), 257-265.

\title{
Boundary Value Problem with Regular Singurarity and Helgason-Okamoto Conjecture
}

by

Katsuhiro MinemurA* and Toshio Oshima**

\section{Part I. Boundary Value Problem with Regular Singularity.}

\section{$\S 1$. The Concept of Boundary Values.}

First we review on the definition of boundary values of solutions of linear differential equations. The most fundamental example is the concept of hyperfunctions, which are the boundary values of holomorphic functions. The space of hyperfunctions on $\boldsymbol{R}$ is defined by $\mathscr{B}(\boldsymbol{R})$ $=\mathcal{O}\left(\boldsymbol{C}_{+}\right) \oplus \mathcal{O}\left(\boldsymbol{C}_{-}\right) / \mathcal{O}(\boldsymbol{C})$. Here $\boldsymbol{C}_{ \pm}=\{z=x+i \boldsymbol{z} \in \boldsymbol{C} ; \pm y>0\}$ and $\mathcal{O}(U)$ is the space of holomorphic functions on a complex manifold $U$. Therefore any hyperfunction $f(x)$ on $\boldsymbol{R}$ equals the sum of the boundary values of solutions of the equation $\bar{\partial}_{z} u(z) \equiv 1 / 2 \cdot(\partial / \partial x+i \partial / \partial y) u(z)=0$ :

$$
f(x)=u_{+}(x+i 0)-u_{-}(x-i 0) \quad\left(u_{ \pm}(z) \in \mathcal{O}\left(\mathbb{C}_{ \pm}\right)\right) .
$$

We may consider that $f(x)$ represents a quantity how the holomorphic function $u=\left(u_{+}, u_{-}\right)(\in \mathcal{O}(\boldsymbol{C}-\boldsymbol{R}))$ can not be extended to a holomorphic function on $\boldsymbol{C}$ without the gap on $\boldsymbol{R}$. But if we don't request to be a solution of the equation $\bar{\partial}_{z} \widetilde{u}=0$, we have an extension $\widetilde{u}(x, y) \quad\left(\in \mathscr{B}\left(\boldsymbol{R}^{2}\right)\right)$ of $u$ because of the flabbiness of the sheaf of hyperfunctions. We set $g(x, y)=\bar{\partial}_{z} \widetilde{u}$, then clearly supp $g \subset \boldsymbol{R}$. The extension $\widetilde{u}$ and $g$ are not uniquely determined by $u$. Considering the relation $2 y \bar{\partial}_{z} h(x) \delta^{(\nu)}(y)$ $=-i(\nu+1) h(x) \delta^{(\nu)}(y)-\nu h^{\prime}(x) \delta^{(\nu-1)}(y)$, we can prove that there exists the unique extension $\widetilde{u}$ of $u$ such that $y \bar{\partial}_{z} \widetilde{u}=0$. In this case we have $g(x, y)=i / 2 \cdot f(x) \delta(y)$. If $u_{ \pm} Y( \pm y)$ is well-defined, this is clear because $\widetilde{u}=u_{+} Y(y)+u_{-} Y(-y)$. The similar prosedure is valid for more general

Received October 7, 1976.

* Department of Mathematics, Japan Women's University.

** Department of Mathematics, Faculty of Science, University of Tokyo. 
boundary value problems.

Let $M$ be an $(n+1)$-dimensional real analytic manifold with a local coordinate system $\left(t, x_{1}, \cdots, x_{n}\right)$. We set $M_{+}=\{t>0\}$ and $N=\{t=0\}$. We can define the boundary values on $N$ of hyperfunction solutions of a differential equation $P\left(t, x, D_{t}, D_{x}\right) u=0$ on $M_{+}$with respect to which $N$ is non-characteristic. (See Komatsu-Kawai [6], Schapira [8]). We explain it by an example. Let $P$ be a differential operator of the form $D_{t}{ }^{2}+A_{1}\left(t, x, D_{x}\right) D_{t}+A_{2}\left(t, x, D_{x}\right)$ of order 2 . Then any hyperfunction solution $u(t, x)$ on $M_{+}$of the equation $P u=0$ has a unique extension $\widetilde{u}(\in \mathscr{B}(M)$, the space of hyperfunctions on $M)$ such that supp $\widetilde{u} \subset \bar{M}_{+}$, $\left.\widetilde{u}\right|_{M_{+}}=u$ and $t^{2} P \widetilde{u}=0$. The boundary values $v_{0}, v_{1}$ of $u$ are defined by $P \widetilde{u}=v_{0}(x) \delta^{\prime}(t)+v_{1}(x) \delta(t)$ and we have $v_{0}=\left.u\right|_{N}, v_{1}=\left.\left(D_{t} u\right)\right|_{N}+A_{1}(0, x$, $\left.D_{x}\right) v_{0}$. Since Holmgren's theorem in hyperfunctions says the map $s p$ : $\left.\left.\mathscr{H}_{\bar{M}_{+}}\left(\mathscr{B}_{M}\right)\right|_{N} \rightarrow \mathscr{C}\right|_{i S_{N^{*}} M}$ is injective, we have the following micro-local expression without the loss of any information:

$$
s p \widetilde{u}=\sum_{j=0}^{1} P^{-1} D_{t}^{2} v_{j}(x) t_{+}^{j} \quad \text { on } \quad i S_{N}^{*} M .
$$

This point of view leads us to the concept of boundary value problems for differential equations with regular singularity.

\section{§ 2. Systems of Differential Equations with Regular Singurarity.}

Let $M$ be an $(l+n)$-dimentional real analytic manifold with a local coordinate system $\left(t_{1}, \cdots, t_{l}, x_{1}, \cdots, x_{n}\right)$. Set $N_{j}=\left\{t_{j}=0\right\}, N^{j}=\left\{t_{i}=0\right.$; $i \neq j\}, N=\left\{t_{1}=\cdots=t_{l}=0\right\}$ and $M_{+}=\left\{t_{1}>0, \cdots, t_{l}>0\right\}$.

Definition 2.1. A system of differential equations on $M$

$$
\mathscr{H}: P_{j} u=0(j=1, \cdots, l)
$$

is said to have regular singularity along the set of the walls $\left\{N_{1}, \cdots, N_{l}\right\}$ if and only if the following conditions hold.

(RS-0) Put $r_{j}=\operatorname{ord} P_{j}$. Then

$$
\left[P_{j}, P_{k}\right]=\sum_{i=1}^{l} G_{j, k}^{i} P_{i}
$$


with differential operators $G_{j, k}^{i}$ satisfying ord $G_{j, k}^{i}<r_{j}+r_{k}-r_{i}$. (RS-1) $P_{j}$ is of the form

$$
P_{j}=P_{j}\left(t, x ; \mathcal{I}_{1}, \cdots, \mathcal{I}_{l}, t D_{x}\right),
$$

where $\mathcal{G}_{j}=t_{j} \partial / \partial t_{j}$ and $t D_{x}=\left(t_{1} D_{x_{1}}, t_{1} D_{x_{2}}, \cdots, t_{l} D_{x_{n}}\right)$.

(RS-2) We denote by $\stackrel{\circ}{p}_{j}(x, s)$ the homogeneous part of $P_{j}\left(0, x, s_{1}, \cdots\right.$, $\left.s_{l}, 0\right)$ of degree $r_{j}$ with respect to $s$. Then $\stackrel{\circ}{p}_{j}(x, s)=0$ for $j=1, \cdots, l$ implies $s=0$ for any point $x \in N$.

In this case, the system of the equations for $s$

$$
P_{j}\left(0, x ; s_{1}, \cdots, s_{l}, 0\right)=0 \quad(j=1, \cdots, l)
$$

has solutions of number $r=r_{1} \times \cdots \times r_{l}$. These solutions are called the characteristic exponents of $\mathscr{M}$. We set them $s^{\nu}(x) \in \mathbb{C}^{l}(\nu=1, \cdots, r)$.

For simplicity we assume that $s^{1}(x)$ is analytic on $N$ and that

$$
s^{1}(x)-s^{\nu}(x) \notin Z_{+}{ }^{l}-\{0\} \equiv\{0,1,2, \cdots\}^{l}-\{0\} \text { for } \nu=2, \cdots, r
$$
and for $x \in N$.

Theorem 2.2. Under the assumption $(A)$, there exists an injective homomorphism of the system

$$
\mathscr{M}_{1}:\left(\mathcal{G}_{j}-s_{j}{ }^{1}(x)\right) u_{1}=0 \quad(j=1, \cdots, l)
$$

to $\mathcal{M}$ micro-locally on $\Lambda=i S^{*} M-\bigcup_{j} i S_{N^{j}}^{*} M$. This homomorphism is given by the correspondence $u_{1}=B\left(t, x, D_{t}, D_{x}\right) u$ with a microdifferential operator $B$.

Let $u(t, x)$ be a hyperfunction solution of $\mathscr{M}$ defined on $M_{+}$. We will define the boundary value on $N$ of the solution under the assumptions (A) and the following (B).

(B) There exists a differential operator $Q_{j}$ in $\mathscr{D}_{M} P_{1}+\cdots+\mathscr{D}_{M} P_{l}$ for $j=1, \cdots, l$, such that the equation $\Re_{j}: Q_{j} w=0$ has a regular singularity in a weak sence along the hypersurface $N_{j}$. Here $\mathscr{D}_{M}$ denotes the ring of differential operators on $M$ and "in a weak sence" means by definition that the transformation $t_{j} \mapsto t_{j}{ }^{m}$ for a large positive integer $m$ makes the equation $\eta_{j}$ have a regular singularity along $N_{j}$. 
Theorem 2.3. Assume $(B)$ and that any characteristic exponent of $\mathscr{\Omega}_{j}$ is not strictly negative integer at any point in $N$. Then any hyperfunction solution $u(t, x)$ of $\mathscr{M}$ defined on $M_{+}$has a unique extension $\widetilde{u}(\in \mathcal{B}(M))$ such that

$$
\operatorname{supp} \widetilde{u} \subset \bar{M}_{+},\left.\widetilde{u}\right|_{M+}=u \quad \text { and } \quad P_{j} \widetilde{u}=0 \quad \text { for } \quad j=1, \cdots, l .
$$

Let $u(t, x)$ be the extension in Theorem 2.3. In the case when a characteristic exponent of $\Re_{j}$ equals a negative integer at a point $x \in N$, we have only to replace $u$ by $t^{k} u$ for a lage positive integer $k$ to apply Theorem 2.3. Then Theorem 2.2 says that

$$
B\left(t, x, D_{t}, D_{x}\right) s p \widetilde{u}=f_{1}(x) t_{+}{ }^{s(x)} \text { on } \Lambda
$$

for a suitable hyperfunction $f_{1}(x)$ on $N$.

Definition 2.4. We call $f_{1}(x) \otimes(d t)^{s^{1}(x)}$ the boundary value of $u(t$, $x$ ) on the edge $N$ with respect to the characteristic exponent $s^{1}(x)$. This hyperfunction valued section of line bundle $\left(T_{N_{1}}^{*} M\right)^{s_{1}{ }^{1}(x)} \otimes \cdots \otimes\left(T_{N_{l}}^{*} M\right)^{s^{s^{1}(x)}}$ on $N$ is defined not depending on the choice of local coordinate system.

For the precise statement and their proof, see [5].

\section{Part II. Application to Helgason-Okamoto Conjecture.}

\section{§ H. Helgason-Okamoto Conjecture.}

Let $D$ be the open unit disc in the complex plane $C, B$ the boundary of $D$ and $\Delta$ the laplacian on $D$ corresponding to the Poincare metric $d s^{2}=(1-z \bar{z})^{-2} d z d \bar{z}(z \in D)$. Then $\Delta$ is given by

$$
\Delta=(1-z \bar{z})^{2} \partial^{2} / \partial z \partial \bar{z} .
$$

Define the (generalized) Poisson kernel $P_{\lambda}(z, b)$ by

$$
P_{\lambda}(z, b)=\left[(1-z \bar{z}) /|z-b|^{2}\right]^{\lambda}, z \in D, b \in B, \lambda \in C .
$$

Then it is easy to see that for any fixed $b, P_{\lambda}(z, b)$ satisfies the following differential equation on $D$

$$
\mathscr{M}_{\lambda}: \Delta P_{\lambda}(z, b)=\lambda(\lambda-1) P_{\lambda}(z, b) .
$$


Theorefore for any hyperfunction $\varphi$ on $B$, the (generalized) Poisson integral

$$
\mathscr{Q}_{\lambda} \varphi(z)=\int_{B} P_{\lambda}(z, b) \varphi(b) d b, d b=d t / 2 \pi, b=\exp (i t),
$$

also satisfies the differential equation $\mathscr{M}_{\lambda}$.

Theorem. (Helgason) The Poisson integrals

$$
\mathscr{Q}_{\lambda} \varphi(z)=\int_{B} P_{\lambda}(z, b) \varphi(b) d b
$$

where $\lambda \in \mathbb{C}$ and $\varphi$ is a hyperfunction on $B$ constitute precisely the eigenfunctions of $\Delta$.

By the way, the above symmetric space $D$ is isomorphic to the symmetric space $X=S U(1,1) / K$, where $K$ is a maximal compact subgroup isomorphic to $S O(2)$, and the differential operator $\Delta$ is a generator of the algebra of $S U(1,1)$-invariant differential operators on $X$. Moreover the Poisson kernel is obtained by the Iwasawa decomposition of $S U(1,1)$ canonically. Theorefore we reformulate the above theorem in view of the group theory.

Let $G$ be a connected real semisimple Lie group with finite center, $K$ a maximal compact subgroup of $G$ and $\mathfrak{g}$ (resp. $\mathfrak{f}$ ) be the Lie algebra of $G($ resp. $K)$. Let $\mathfrak{p}$ be the orthogonal complement of $\mathfrak{l}$ in $\mathfrak{g}$ with respect to the Killing form of $\mathfrak{g}$ and $\mathfrak{a}$ be a maximal abelian subspace of p. Let $M$ (resp. $M^{\prime}$ ) be the centralizer (resp. normalizer) of $A$ in $K$ and put $W=M^{\prime} / M$. Let $\Sigma$ be the set of roots with respect to ( $g$, a) and fix a lexicographic order on $\mathfrak{a}^{*}$, the real dual space of $\mathfrak{a}$. Let $\Sigma_{+}$be the set of positive roots, $\left\{\alpha_{1}, \cdots, \alpha_{l}\right\}$ the set of simple roots $(l=\operatorname{dim} \mathfrak{a}), g_{\alpha}$ the root space corresponding to the root $\alpha$ and $\rho$ the half sum of the positive roots with multiplicity. Let $\mathfrak{n}=\sum_{\alpha \in \Sigma_{+}} \mathfrak{g}_{\alpha}$ and let $A$ (resp. $N$ ) denote the analytic subgroup of $G$ corresponding to $\mathfrak{a}$ (resp. $\mathfrak{n}$ ). Then $G=K A N$ (Iwasawa decomposition). For any $g$ in $G$, we write $H(g)$ for the unique element $H$ in a such that $g=k(\exp H) n$ where $k \in K$ and $n \in N$. Put $X=G / K, B=K / M$ and let $\boldsymbol{D}(X)$ denote the algebra of $G$-invariant differential operators on $X$. 
For any $\lambda$ in $\mathfrak{a}_{\boldsymbol{C}}^{*}$ (complex dual space of $\mathfrak{a}$ ), put

$$
\Phi_{\lambda}(g K)=\int_{K} \exp \{\lambda(H(g k))\} d k
$$

where $d k$ is the normalized Haar measure on $K$. Then $\Phi_{\lambda}$ is a spherical function and is a joint eigenfunction of $\Delta$ in $D(X)$. Let $\chi_{\lambda}$ be the unique algebra homomorphism of $\mathbb{D}(X)$ into $\mathbb{C}$ (character of $\boldsymbol{D}(X)$ ) such that $\Delta \Phi_{\lambda}=\chi_{\lambda}(\Delta) \Phi_{\lambda}(\Delta \in \mathbb{D}(X))$.

For any character $\chi$ of $\mathbb{C}(X)$, let $\mathcal{A}(X, \mathcal{M}(\chi))$ denote the space of real analytic functions on $X$ satisfying the following system of differential equations

$$
\mathscr{M}(\chi): \Delta u=\chi(\Delta) u, \Delta \in \mathbb{D}(X) .
$$

For $\lambda$ in $\mathfrak{a}_{C}^{*}$, let $P_{\lambda}(x, b)$ be the real analytic function on $X \times B$ defined by

$$
P_{\lambda}(g K, k M)=\exp \left\{-(\lambda+2 \rho)\left(H\left(g^{-1} k\right)\right)\right\}, g \in G, k \in K .
$$

Then for any fixed $b$ in $B, P_{\lambda}(x, b)$ belongs to $\mathcal{A}\left(X, \mathcal{M}\left(\chi_{\lambda}\right)\right)$.

Let $d b$ be the $K$-invariant normalized measure on $B$. For any hyperfunction $\varphi$ on $B$, we define the Poisson integral $\mathscr{L}_{\lambda} \varphi$ on $X$ by

$$
\mathscr{Q}_{\lambda} \varphi(x)=\int_{B} P_{\lambda}(x, b) \varphi(b) d b .
$$

Conjecture. (Helgason-Okamoto) Poisson integrals

$$
\mathscr{L}_{\lambda} \varphi(x)=\int_{B} P_{\lambda}(x, b) \varphi(b) d b
$$

where $\lambda \in \mathrm{a}_{\boldsymbol{C}}^{*}$ and $\varphi$ is a hyperfunction on $B$ constitute the joint eigenfunction of $\Delta$ in $\mathbb{D}(X)$.

\section{§4. Approach to the Helgasom-Okamoto Conjecture by the Method of Fourier Seroies Expansion.}

On the Helgason-Okamoto conjecture, there have appeared several papers [1], [3], [7] dealing with the conjecture in the case that the rank of the symmetric space is equal to one. The proofs given in these papers follow after the idea due to $\mathrm{S}$. Helgason. We review the idea 
in the case of the unit disc. For details, see Helgason [2, Chap. IV].

Since the compact Lie group $K=S O(2)$ acts on the unit disc $D$ as linear fractional transtormations, any eigenfunction $u$ of $\Delta$ can be expanded in a Fourier series

$$
u(z)=\sum_{n=-\infty}^{\infty} u_{n}(z)
$$

where

$$
u_{n}(z)=\frac{1}{2 \pi} \int_{0}^{2 \pi} u\left(e^{i t} z\right) e^{-i n t} d t
$$

Put $\varphi_{n}\left(e^{i t}\right)=e^{i n t}$ and define the function $u_{n}{ }^{2}$ by

$$
u_{n}^{\lambda}(z)=\mathscr{L}_{\lambda} \varphi_{n}(z)=\frac{1}{2 \pi} \int_{0}^{2 \pi} P_{\lambda}\left(z, e^{i t}\right) e^{i n t} d t
$$

Let $V_{n}{ }^{\mu}$ be the subspace of $\mathcal{A}\left(X, \mathcal{M}_{\mu}\right)$ consisting of the elements $u$ with the property that $u\left(e^{i t} z\right)=e^{i n t} u(z)$. Then it is easy to see that $u_{n}$ and $u_{n}{ }^{2}$ both belong to $V_{n}^{\lambda(\lambda-1)}$. It is known by the representation theory that $\operatorname{dim} V_{n}{ }^{\mu}$ is equal to one. By solving the differential equation of one variable which $u_{n}^{2}$ satisfies, we have

$$
u_{n}^{\lambda}(r)=\left(1-r^{2}\right) r^{|n|} \frac{\Gamma(|n|+\lambda)}{\Gamma(\lambda) \Gamma(|n|+1)} F\left(\lambda,|n|+1,|n|+1 ; r^{2}\right) \quad(0 \leqq r<1) .
$$

Hence, if $\lambda \neq 0,-1,-2, \cdots$, then from the fact that $\operatorname{dim} V_{n}^{\lambda(\lambda-1)}=1$, it follows that $u_{n}$ is a constant multiple of $u_{n}{ }^{2}$ and $u$ is expanded as

$$
u=\sum_{n=-\infty}^{\infty} a_{n} u_{n}^{\lambda}=\sum_{n=-\infty}^{\infty} a_{n} \mathcal{L}_{\lambda}\left(\varphi_{n}\right)
$$

Using the asymptotic behavior of the function $u_{n}{ }^{2}$ near the boundary, we have an estimate on the growth order of the sequence $\left(a_{n}\right)$;

$$
\sum_{n=-\infty}^{\infty}\left|a_{n}\right| r^{|n|}<\infty \quad(0 \leqq r<1),
$$

which assures that the sequence $\varphi=\sum_{n=-\infty}^{\infty} a_{n} \varphi_{n}$ defines a hyperfunction on $B$. It is easy to see that the Poisson integral of $\varphi$ coincides with the given eigenfunction $u$.

The method explained above was used to prove the Helgason-Okamoto conjecture in some series of symmetric spaces of rank one. But if the rank of the symmetric space is higher, the decomposition of the space of eigenfunctions as a sum of irresucible $K$ modules is very complicated, and moreover one encounters the crucial difficulty that the radial 
components of invariant differential operators are not ordinary differential operators so that one can not apply the classical results.

\section{$\S 5$. Approach to the Helgason-Okamoto Conjecture by the Theory of the System of Differential Equations with Regular Singularity.}

In the meanwhile, as is stated in Part I, the notion of "regular singularity" for the ordinary differential equation is generalized to that for the system of partial differential equations. It is essential that the system of invariant differential equations on a symmetric space has regular singularity along the Martin boundary $B$, which assures the existence of boundary values as hyperfunctions. It depends on how to imbed the symmetric space whether the system of invariant differential equations has regular singularity along the Martin boundary. In a higher rank case, the Martin boundary does not appear in the usual imbedding. Here we explain our imbedding in which the Martin boundary appears.

Let $X^{\prime}$ be the subset of regular elements in $X$. Then $X^{\prime}$ is an open dense submanifold and is real-analytically isomorphic to $\Omega_{+}=B \times(0,1)^{l}$, where $l$ is the rank of $X$. Put $\Omega=B \times(-1,1)^{l}$ and regard $\Omega_{+}$as an open submanifold of $\Omega$ canonically. Let $N_{i}(i=1, \cdots, l)$ be the hypersurfaces defined by

$$
N_{i}=\left\{(b, y) \in \Omega ; y=\left(y_{1}, \cdots, y_{l}\right), y_{i}=0\right\} .
$$

Proposition 5. 1. Every invariant differential operator $\Delta$ in $\boldsymbol{D}(X)$ is analytically extended to a differential operator on $\Omega$. The system $\mathscr{M}(\chi)$ has regular singularity along the walls $N_{i}(i=1, \cdots, l)$ and satisfies Assumption (B).

Let $H_{i}(i=1, \cdots, l)$ be the dual basis for $\mathfrak{a}$ with respect to the simple roots $\alpha_{i}(i=1, \cdots, l)$. For $w \in W$ and $\lambda \in \mathfrak{a}_{\boldsymbol{C}}^{*}$, put $\lambda^{w}=w(\lambda+\rho)$ $-\rho$ and $\lambda(w)=\left(-\lambda^{w}\left(H_{1}\right), \cdots,-\lambda^{w}\left(H_{l}\right)\right)$. Then the set of characteristic exponents is given by $\{\lambda(w) ; w \in W\}$ and Assumption (A) for $\lambda(1)$ ( 1 is the identity element in $W$ ) is equivalent to the assumption

$(\mathrm{A})^{\prime} \quad-\frac{2\langle\lambda+\rho, \alpha\rangle}{\langle\alpha, \alpha\rangle} \notin \boldsymbol{Z}_{+}-\{0\} \quad$ for any $\alpha$ in $\Sigma_{+}$ 
where $\langle\cdot, \cdot\rangle$ is the inner product induced by the Killing form of $\mathfrak{g}$ and $\boldsymbol{Z}_{+}$is the set of non-negative integers. Hereafter we assume Assumption (A)'.

Now we consider the system $\mathscr{M}\left(\chi_{\lambda}\right)$. For any $u$ in $\mathscr{A}\left(X, \mathscr{M}\left(\chi_{\lambda}\right)\right)$ we can define the boundary value $\beta_{\lambda}(u)$ on $B$ with respect to the characteristic exponent $\lambda(1)$. By the aid of the theory of spherical functions of Harish-Chandra, it is proved that the map $\beta_{k}$ is an inverse of the Poisson integral up to a constant multiple. Thus we obtain the main

Theorem 5.2. Under Assumption $(A)^{\prime}$, the Poisson integral $\mathscr{P}_{\lambda}$ is a topological isomorphism between Fréchet-Schwartz spaces $\mathcal{B}(B)$ and $\mathcal{A}\left(X, \mathcal{M}\left(\chi_{2}\right)\right)$.*)

Corollary 5.3. For any character $\chi$ of $\mathbb{D}(X)$, we can find $a \lambda$ in $\mathfrak{a}_{\boldsymbol{C}}^{*}$ such that $\mathscr{Q}_{\lambda}$ is a topological isomorphism of $\mathscr{B}(B)$ onto $\mathcal{A}(X$, $\mathscr{M}(\chi))$.

\section{References}

[1] M. Hashizume, K. Mineura and K. Okamoto, Harmonic functions on hermitian hyperbolic spaces, Hiroshima Math. J. 3 (1973), 81-108.

[2] S. Helgason, A duality for symmetric spaces with applications to group representations, Advances in Math. 5 (1970), 1-154.

[3] S. Helgason, Eigenspaces of the Laplacian; Integral Representations and Irreducibility, J. Functional Analysis 17 (1974), 328-353.

[4] M. Kashiwara, A. Kowata, K. Minemura, K. Okamoto, T. Oshima and M. Tanaka, Eigenfunctions of invariant differential operators on a symmetric space, to appear.

[5] M. Kashiwara and T. Oshima, Systems of differential equations with regular singularity and their boundary value problem, to appear.

[6] H. Komatsu and T. Kawai, Boundary values of hyperfunction solutions of linear partial differential equations, Publ. Res. Inst. Math. Sci., 7 (1971), 95-104.

[7] K. Minemura, Eigenfunctions of the laplacian on a hermitian hyperbolic space, Hiroshima Math. J. 4 (1974), 441-457.

[8] P. Schapira, Problème de Dirichlet et solutions des hyperfunctions des équations elliptiques, Bull. UMI (4), 3 (1969), 367-372.

*) Here the topology of $\mathcal{A}\left(X, \mathscr{M}\left(\chi_{2}\right)\right)$ is induced by the natural topology of $C^{\infty}(X)$, 
